\title{
Rut Diamint
}

\section{Streitkräfte und Demokratie}

\section{Einleitung}

Mitte der 1980er Jahre fanden in Argentinien drei Erhebungen der Streitkräfte statt, die trotz ihres beschränkten Erfolges Zweifel hinsichtlich der Konsolidierung der Demokratie und der Möglichkeit eines erneuten Staatsstreiches aufkommen ließen. Der vorerst letzte Aufstand fand 1990 statt, wurde rasch gewaltsam unterdrückt und hinterließ ein Saldo von mehreren Toten. Seitdem nahm die Überzeugung zu, dass die Nation damit den Zyklus von Staatsstreichen überwunden habe. Die Spielräume der Streitkräfte wurden eingeschränkt, sie sind nicht dazu in der Lage, die allgemeine politische Tagesordnung gemäß ihren Interessen zu gestalten und können auch in sicherheitspolitischen Fragen keinen dominierenden Einfluss ausüben. Trotzdem bedeutet dies weder, dass autoritäre Konzeptionen vollständig aus den militärischen Institutionen verschwunden wären, noch dass die Streitkräfte sich durch tiefe Überzeugungen im Sinne einer Verteidigung der Demokratie auszeichnen würden.

Bei den folgenden Betrachtungen wird sich zeigen, dass sich die Streitkräfte nach wie vor in die nationale Politik einmischen. Zwar haben die Streitkräfte die Spielregeln der Demokratie akzeptiert, sie halten sich selbst als politischen Akteur jedoch nicht für völlig unentbehrlich. Diese Haltung ist insofern beunruhigend, als sie an die in autoritären Phasen kultivierten Praktiken anknüpft und der Ungewissheit über die demokratischen Überzeugungen der Militärs neue Nahrung gibt. Ein Staatsstreich ist allerdings deswegen nicht am Horizont auszumachen. Dies hängt auch mit den globalen Demokratisierungstendenzen, mit den durchgeführten Staatsreformen im Sinne von Modernisierung und Marktöffnung sowie mit den Auswirkungen beständigen Drucks von Seiten der Zivilgesellschaft zusammen. Zweifellos wurden bei der Demilitarisierung der argentinischen Gesellschaft Fortschritte gemacht, auch wenn ein Vergleich der Realität mit theoretischen Konzepten von Autoren wie Huntington und Stepan bedeutende Schwächen im Hinblick auf das Idealmodell ziviler Suprematie belegt.

Die Transformation der Rolle der Streitkräfte ist auch im Zusammenhang mit deren Scheitern bei der Führung des Staates sowie auf mili- 
tärischer Ebene zu sehen. Hinzu kommen externe Faktoren wie die Unsicherheiten nach dem Ende des Kalten Krieges und interne Aspekte wie die Auswirkungen der Kürzungen im Militärhaushalt. Zur Mission der Streitkräfte gehört es nicht mehr, Wächter der Nation gegenüber einem hypothetischen Vordringen des Kommunismus im Inneren zu sein. Auch im Hinblick auf die Landesverteidigung gegenüber externen Bedrohungen hat sich die Situation grundlegend verändert, denn die Gegner von einst sind heute Partner im Rahmen eines integrierten Marktes.

Allerdings bleiben einige Fragen offen, beispielsweise: Welche Auswirkungen haben die nicht eliminierten Vorrechte der Militärs auf die demokratischen Institutionen? Welcher Strategien bedienen sich die Streitkräfte, um zumindest einen Teil ihrer Macht zu bewahren? Welche Haltung würden die Offiziere im Falle einer Krise der Demokratie einnehmen? Beeinträchtigt die Identitätskrise der Streitkräfte die Stabilität der Demokratie? Vielleicht müssen wir erkennen, dass die demokratischen Gesellschaften trotz des Fortbestehens einer wenig liberalen und toleranten politischen Kultur in vielen Ländern Lateinamerikas selbst dann Fortschritte verzeichnen können, wenn weiterhin Praktiken existieren, die gegen das Regime gewandt sind.

Selbst in Ländern, in denen die Militärs noch über beachtliche Vorrechte verfügen, ist es der Demokratie gelungen, Fortschritte auf Gebieten zu erzielen, die von den Streitkräften mit besonderer Aufmerksamkeit beobachtet werden; dies betrifft u.a. die Anklagen wegen Menschenrechtsverletzungen, Kürzungen in den Militärhaushalten, die Partizipation der Parlamente bei Fragen der Verteidigungspolitik und die Absetzung von führenden militärischen Amtsträgern (Diamint 1999). Solche Entscheidungen wurden von den argentinischen Zivilregierungen getroffen und sprechen für einen Strategiewechsel der herrschenden Eliten, die nicht länger als Verbündete der Streitkräfte angesehen werden können. Im Verlauf des vergangenen Jahrzehnts hat sich in Argentinien eine neue politische Matrix herausgebildet, eine neue Form der wechselseitigen Beziehungen zwischen Akteuren und Institutionen (Acuña 1995). Zur Beilegung von Interessenkonflikten zwischen den verschiedenen Akteuren gelten heutzutage die Spielregeln der Demokratie und nicht Garantien von Seiten der Militärs. Angesichts dieser neuen Konstellation mussten auch die Streitkräfte nach neuen Formen suchen, um sich einen gewissen Grad der Einflussnahme auf das politische und soziale Geschehen zu bewahren. Ihre erste Priorität gilt dabei der Verteidigung der eigenen Institution (Zagorski 1992). 
Im folgenden Abschnitt wird geschildert, wie man in Argentinien während des Übergangs zur Demokratie mit dem Thema Streitkräfte umgegangen ist. Anschließend wird die Strategie der Regierung Menem gegenüber den Streitkräften untersucht. Im dritten Abschnitt erfolgt eine Analyse der militärpolitischen Entscheidungsprozesse. Der vierte Abschnitt bezieht sich auf die zivil-militärischen Beziehungen. Als fünfter Aspekt wird die Verteidigungspolitik analysiert.

\section{Der Umgang mit den Streitkräften unter Alfonsín}

Die letzte Militärdiktatur (1976-1983) hatte sich selbst als „Prozess der nationalen Reorganisation" bezeichnet und war mit dem Anspruch angetreten, die „Ordnung wiederherzustellen“", doch als sich die Militärs 1983 in die Kasernen zurückziehen mussten, waren sie sowohl politisch als auch militärisch auf der ganzen Linie gescheitert. Der Anfang vom Ende der Diktatur begann mit dem verhängnisvollen Fehler, sich auf eine kriegerische Auseinandersetzung mit der wichtigsten Militärmacht Westeuropas einzulassen und machte deutlich, wie sehr sich die Militärs hinsichtlich der internen und externen politischen Situation geirrt hatten. Die Bilanz der Diktatur war aber auch sonst in jeder Hinsicht katastrophal. Eine zerrüttete Verwaltung, das moralische Versagen, die Erhöhung der Auslandsschulden von 7 Milliarden US\$ (1976) auf 44 Milliarden US\$ (1984), die Verstöße gegen die Menschenrechte, die heftigen Angriffe gegen die Rechtsordnung, dies sind einige Beispiele für das Erbe der Diktatur, die erklären können, warum die Rolle der Streitkräfte von der argentinischen Gesellschaft grundlegend in Frage gestellt wurde. Die Militärs sahen sich mit einer ganzen Palette von Vorwürfen konfrontiert: Korruption, Bürokratisierung, Ineffizienz und Disziplinlosigkeit. Nach der Niederlage gegen Großbritannien blieb den Streitkräften ein Jahr, um ihren Rückzug in die Kasernen zu organisieren. Die Streitkräfte waren besiegt worden, aber nicht vernichtet, weshalb es ihnen auch während der Regierungszeit von Raúl Alfonsín wiederholt gelang, ihren korporativen Interessen Gehör zu verschaffen.

Sieben Jahre Militärdiktatur hatten in vielerlei Hinsicht ihre Spuren hinterlassen, von den politischen Parteien, den Gewerkschaften und Berufsverbänden bis hin zu den Universitäten und den Praktiken des täglichen Zusammenlebens (Rouquié 1988). Die neue zivile Regierung musste nicht nur ein zerrüttetes Land verwalten, sondern gleichzeitig die Rekonstruktion eines sozialen Geflechts unterstützen, dessen Institutionen stark in Mitleidenschaft gezogen waren. Die Regierung Alfonsín (1983- 
1989) tat sich schwer damit, eine kohärente und entschiedene Politik in Militärfragen zu verfolgen. Die Zivilgesellschaft verfügte ihrerseits nicht über die notwendigen Kräfte und Ressourcen, um sich an der Lösung der entsprechenden Probleme zu beteiligen. Die Regierung war nicht da$\mathrm{zu}$ in der Lage, den Konflikt mit den Streitkräften zu lösen und noch weniger, eine zwischen Militärs und Zivilisten abgestimmte Verteidigungspolitik auf den Weg zu bringen.

Alfonsín begann sein Mandat mit zwei Dekreten, die bereits während des Präsidentschaftswahlkampfes angekündigt worden waren: Am 12. Dezember 1983 wurden gerichtliche Untersuchungen gegen die führenden Köpfe der in den 1970er Jahren aktiven Guerillaorganisationen angeordnet (Dekret No. 157), einen Tag später Untersuchungen gegen die Mitglieder der drei Militärjuntas, die seit 1976 an der Macht gewesen waren (Dekret No. 158). Gleichzeitig legte der Präsident dem Parlament einen Gesetzentwurf vor, der die Außerkraftsetzung und Annullierung der von den Militärs im September 1983 verkündeten Selbstamnestie vorsah. ${ }^{1}$

Im Zentrum der Militärpolitik der UCR-Regierung stand die Stärkung des Verteidigungsministeriums und die Verabschiedung eines neuen Verteidigungsgesetzes. Das Ministerium sollte eine effektive Kontrolle sowohl über die Führung der Streitkräfte als auch hinsichtlich des Militärhaushaltes, der Gehälter und der Produktion von Rüstungsgütern ausüben. Ein anderes Element, mit dessen Hilfe die Legitimität der Streitkräfte erneuert werden sollte, war die Militärreform. „Die Militärreform [...] muss einen neuen moralischen Ton im Rahmen des vollständigen Respekts gegenüber der verfassungsmäßigen Ordnung gewährleisten ", sagte Präsident Alfonsín in einer Rede vor der Kameradschaft der Streitkräfte aus Anlass der Feierlichkeiten zum Unabhängigkeitstag am 9. Juli 1985. Er fügte hinzu:

Statt Verteidiger der nationalen Gemeinschaft zu sein, haben sich die Streitkräfte in deren Führer und Verwalter verwandelt, was einer Negation des entscheidenden Kerns der Rolle der Streitkräfte in einer zivilisierten, modernen und komplexen Nation entspricht [...]. (zitiert nach Clarin, 6. Juli 1985: 3)

1 Das als „Gesetz zur nationalen Befriedung“ bekannte Dekret 22.924 vom 22. September 1983 stellte den letzten Versuch der Streitkräfte dar, die zukünftige verfassungsmäßige Regierung auf eine ihren Interessen gemäße Politik festzulegen. 
Der Grundtenor dieser Kritik verdeutlicht den konfliktiven Stil, durch den sich die Beziehungen zwischen Streitkräften und Regierung nach dem Übergang zur Demokratie auszeichneten. Um diese Beziehungen auf den rechten Weg zu bringen, setzte die Regierung auf folgende Maßnahmen:

1.) Abschaffung der Posten der Oberkommandierenden der drei Teilstreitkräfte, wodurch der Generalstabschef zum höchstrangigen Offizier aufgewertet wurde;

2.) Etablierung des Verteidigungsministeriums als politische Führungsinstanz der Streitkräfte;

3.) Umwandlung des Gemeinsamen Generalstabs in ein Organ zur Integration und Zentralisierung der Teilstreitkräfte;

4.) Reform der Militärrechtsordnung und des zivilen Strafprozessrechtes, die v.a. Berufungen gegen von Militärtribunalen ausgesprochene Urteile vor den Zivilgerichten ermöglichen sollte. Mit dem im Februar 1984 verabschiedeten Gesetz 23.049 wurde der Tätigkeitsbereich der zivilen Bundesgerichte als Appellationskammer ausgeweitet. In erster Instanz behielt jedoch die Militärjustiz ihre Zuständigkeit. Die Regierung verfolgte die Idee eines Selbstreinigungsprozesses innerhalb der Streitkräfte. Dies setzte aber voraus, dass diese die Illegitimität der im Kampf gegen die Subversion angewandten Mittel anerkennen würden. Die Reaktion der Militärgerichte entsprach diesen Erwartungen ganz und gar nicht.

5.) Einrichtung gemeinsamer Regionalkommandos, um die Zusammenarbeit zwischen den Waffengattungen zu verbessern.

Die Reformvorschläge trafen auf diverse Schwierigkeiten. Dazu gehörten die wirtschaftlichen Probleme, das Fehlen von Humanressourcen zur Verwirklichung des Wandels, vor allem aber der Widerstand von Seiten der Streitkräfte, die nicht dazu bereit waren, die Legitimität der zivilen Kontrolle anzuerkennen.

Mit dem Präsidialdekret 187/83 wurde die CONADEP geschaffen, eine Kommission zur Untersuchung des Verschwindens von Personen während der Diktatur. Ihr gehörten anerkannte Persönlichkeiten des öffentlichen Lebens sowie aus unterschiedlichen parteipolitischen Richtungen an. ${ }^{2}$ Die Exekutive vertrat die Ansicht, dass , die Menschenrechtsfra-

2 Es handelt sich um Ricardo Colombres, René Favaloro, Hilario Fernández Long, Carlos G. Gattinoni, Gregorio Klimovsky, Marshall Meyer, Jaime F. de Nevares, Eduardo Rabossi, Magdalena Ruiz Guiñazú und Ernesto Sábato. 
ge nicht nur die öffentlichen Institutionen angeht, sondern die Zivilgesellschaft und die internationale Gemeinschaft betrifft", weshalb sie nicht dazu bereit war, der Forderung nach Einrichtung einer parlamentarischen Untersuchungskommission zu entsprechen. In der Tat fürchtete die Regierung die parteipolitische Instrumentalisierung einer derartigen Kommission.

Die Streitkräfte zeigten ein korporatives Verhalten und sahen sich durch die Regierung herausgefordert, die sie global als Feind qualifizierten. Das Gefühl, von der Gesellschaft angegriffen zu werden, verstärkte sich, als im April 1985 der öffentliche Prozess gegen die ehemaligen Kommandanten der Militärregierung begann. Die Kluft zwischen Zivilisten und Militärs vertiefte sich, während die Regierungspartei die Verabschiedung eines Verteidigungsgesetzes verzögerte, das als Rahmen für die zivil-militärischen Beziehungen dienen sollte. Unerklärlicherweise wurde nicht versucht, sich auf eine gemeinsame Strategie mit der Opposition zu einigen. Die Regierungspolitiken, welche die Verantwortlichen der Militärdiktatur zur Rechenschaft ziehen sollten, führten zur Schwächung der Institution Militär als solche und provozierten unerwünschte Effekte. Die Militärs konnten - und wollten - nicht die Vorzüge einer konstitutionellen Demokratie erkennen sowie der Möglichkeit, sich ohne politische Verpflichtungen zu professionalisieren, auch wenn sie durchaus ihre Defizite im Hinblick auf die Verteidigungsaufgabe anerkannten.

Die übereilte Antwort der Regierung auf die Spannungen bestand darin, „eine Situation der Rechtsunsicherheit zu beenden, was zur Befriedung der Gemüter und zur Sicherung des Zusammenlebens zwischen allen Argentiniern beitragen" werde; per Gesetz schränkte sie den Zeitraum für die Aufnahme neuer Prozesse wegen Menschenrechtsverletzungen während der Diktatur stark ein (Ley de Punto Final; ,Schlusspunktgesetz"). ${ }^{3}$ Es blieben lediglich zwei Monate Zeit, um alle Anklagen wegen Delikten im Zusammenhang mit dem Kampf gegen die Subversion zu erheben. Nach Ansicht der Verfechter des Gesetzes würden sich dadurch die Gemüter der Streitkräfte beruhigen lassen. Gleichzeitig verbliebe der Bevölkerung noch eine gewisse Zeit, um Anzeigen gegen die Militärs einzureichen. In einer verantwortlichen und für die Streitkräfte überraschenden Art und Weise bemühten sich die Bundesgerichte jedoch darum, innerhalb des durch das Gesetz konzedierten Zeitraumes

3 Dieses Gesetz (23.492) verfügte die Einstellung der Strafverfolgung gegen jede Person, die nicht innerhalb von 60 Tagen nach Inkrafttreten des Gesetzes durch ein zuständiges Gericht zur Vernehmung vorgeladen wurde. 
alle Militärs, gegen die Verdachtsmomente vorlagen, gerichtlich vorzuladen. Damit war die Hoffnung der Regierung hinfällig, mit dem Schlusspunktgesetz könne man die Ruhe innerhalb der Streitkräfte wiederherstellen. Die Flut von innerhalb kürzester Zeit auf den Weg gebrachten Strafverfahren führte eher noch zu einer Verstärkung der Unruhe.

Dieses Klima gab den Rahmen ab für ein anderes Phänomen, das der ersten post-diktatorialen Regierung großes Kopfzerbrechen bereitete: die militärischen Erhebungen. Der erste Aufstand fand in der Osterwoche 1987 durch eine Gruppe von Offizieren unter Führung von Oberstleutnant Aldo Rico statt. Er begann mit der Weigerung des Majors Ernesto Barreiro, der Vorladung durch das Bundesgericht mit Sitz in Córdoba Folge zu leisten. Die Aufständischen selbst bezeichneten ihr Handeln als "Operation Würde“ und betrachteten es als ihre Mission, die Ehre wiederzuerlangen und die Disziplin wiederherzustellen. Sie sahen sich als Helden des Malvinas-Krieges und als Märtyrer des Kampfes gegen die Subversion. Sie bildeten eine Gruppe, die gegen das System, d.h. gegen das republikanische und demokratische Regime agierte und sich durch stark nationalistische und fundamentalistische Tendenzen auszeichnete.

Im Mai 1987 wurde ein zweites auf Initiative der Regierung erarbeitetes Gesetz mit Konsequenzen für die juristische Aufarbeitung der Menschenrechtsverletzungen während der Diktatur verabschiedet, das sogenannte „Gesetz über die Gehorsamspflicht" (Ley de Obediencia Debida; Gesetz 23.521). ${ }^{4}$ Damit sank die Anzahl der Prozesse wegen Menschenrechtsverletzungen, die sich infolge des Schlusspunktgesetzes bereits auf 450 reduziert hatte, weiter, und zwar auf etwa 100 Fälle. Im Verlauf der Verhöre ging die Zahl auf 20 Fälle zurück und zuletzt blieben nur noch 18 übrig.

Im April 1988 wurde nach langen Verhandlungen und Debatten mit den Militärs und ihren zivilen Verbündeten das Verteidigungsgesetz (Gesetz 23.554) verabschiedet. Damit wurden die Aufgaben der Streitkräfte bei der Landesverteidigung definiert und ihre Partizipation bei Fragen

4 In Artikel 1 des Gesetzes heißt es: ,[...] können nicht strafrechtlich verfolgt werden wegen Delikten, auf die sich Art. 10, Absatz 1 des Gesetzes 23.049 bezieht, weil sie in Ausübung der Gehorsamspflicht gehandelt haben. [...] In solchen Fällen wird es als vollständig mit dem Recht übereinstimmend betrachtet, dass die genannten Personen in einer Zwangssituation bei Unterordnung unter eine übergeordnete Autorität und in Erfüllung von Befehlen operierten, ohne die Fähigkeit oder die Möglichkeit zur Untersuchung, der Opposition oder des Widerstandes gegen diese, hinsichtlich ihrer Angemessenheit oder Legitimität. ${ }^{c}$ 
der inneren Sicherheit eingeschränkt. Ein Nationaler Verteidigungsrat (Consejo de Defensa Nacional, CODENA) unter Vorsitz des Staatspräsidenten wurde eingerichtet. Die Aufgaben des Gemeinsamen Generalstabs wurden neu definiert. Es wurde festgelegt, dass binnengerichtete nachrichtendienstliche Tätigkeiten keine Aufgabe der militärischen Einrichtungen sein durften.

Paradoxerweise wurden trotz der Konfrontation zwischen Regierung und Streitkräften in dieser Phase Pläne für eine strategische Bewaffnung entwickelt. Am Beispiel des Raketenprojekts Condor II, welches mit geheimen Fonds, deutscher Technologie und finanziert durch arabische Länder vorangetrieben wurde, zeigt sich, dass es konkrete Planungen für die Streitkräfte gab. Die Regierung betrachtete es als notwendig, mit irgendeinem Sektor der Streitkräfte ein Bündnis einzugehen. Der Luftwaffe fiel dabei eine privilegierte Rolle zu. Sie war von allen Waffengattungen im besten Zustand und sah sich den geringsten Anschuldigungen ausgesetzt. Weniger in Frage gestellt hinsichtlich des Kampfes gegen die Subversion und erfolgreicher im Malvinen-Krieg, konnte sich die Luftwaffe vom negativen Image der anderen Teilstreitkräfte abgrenzen und es gelang ihr, ihre Bedeutung innerhalb der Militärstruktur zu erhöhen.

Bemüht man sich um eine Bilanz der Verteidigungspolitik der UCRRegierung, so sind auf der Habenseite zu verbuchen: die Aufwertung des Gemeinsamen Generalstabs, die Übertragung des militärisch-industriellen Komplexes an das Verteidigungsministerium, die einheitliche Gestaltung des Militärhaushaltes, die Übertragung der Funktion des Oberkommandierenden auf den Staatspräsidenten. Dazu kamen weitere Maßnahmen, die nicht vollständig umgesetzt werden konnten, beispielsweise die Umstrukturierung von Grenz- und Wasserschutzpolizei sowie deren Beziehungen zu den drei Teilstreitkräften. Auch im Bereich der militärischen Ausbildung wurden einige Veränderungen im Sinne einer Öffnung gegenüber den zivilen Institutionen angestoßen. Auf der Sollseite kommt man jedoch nicht umhin zu betonen, dass eine transparente Verhandlungspolitik fehlte, die zur Überwindung der wechselseitigen Feindseligkeiten beigetragen und zu einer Debatte unter Einschluss der gesamten Gesellschaft geführt hätte.

Die von Alain Rouquié 1988 gezogene Bilanz richtet den Blick auf eine weitere Konsequenz der Übergangsphase:

Ich habe den Eindruck, dass die Militärs aufgrund dessen, was sie in der Osterwoche, in Monte Caseros, etc. erlebt haben und auch wenn Nostalgien hinsichtlich einer starken Macht fortbestehen (und das ist in allen Gesell- 
schaften so, egal wie stabil und demokratisch sie auch sein mögen), keine Anziehungskraft mehr auf bedeutende zivile Sektoren ausüben, wie dies vor 1976 der Fall war, und man spürt mehr Bewusstsein dafür, dass die Institutionen verteidigt werden müssen, denn alle, aus allen politischen Parteien, aus allen Sektoren, aus allen Ideologien sind sich darüber klar geworden, dass es keine guten Diktaturen gibt. Ich glaube, dass dies ein kultureller Wandel ist. (zitiert nach Página 12, 16. 11.1988: 10)

Als Carlos Menem 1989 die Regierungsgeschäfte übernahm, waren die später erfolgten Veränderungen im Bereich der zivil-militärischen Beziehungen in keiner Weise absehbar. Ausgehend von einer Kontinuitätslinie zwischen dem historischen Peronismus, der aus den Reihen der Streitkräfte hervorgegangen war, und den Vorwürfen eines Paktes zwischen Gewerkschaften und Streitkräften, die der spätere Präsident Alfonsín im Wahlkampf 1983 gegen den Peronismus erhoben hatte, konnte man damit rechnen, dass die Militärs in der neuen peronistischen Regierung über einen Verbündeten für die Verwirklichung ihrer Vorstellungen verfügen würden. Doch obwohl eine der ersten Maßnahmen von Präsident Menem in der Begnadigung zahlreicher wegen Menschenrechtsverletzungen verurteilter Militärs bestand, veränderten sich die Beziehungen zwischen Zivilgesellschaft und Streitkräften beträchtlich. Mit der Niederwerfung des letzten Carapintada-Aufstandes ergab sich eine Wende, die zum Teil auf interne Faktoren zurückzuführen war, wie Acuña/Smulovitz (1995: 103-107) betonen:

Der wiederholte Bruch der Befehlskette sowie die Tatsache, dass die Unterstützung für die Anführer der Carapintadas zum größten Teil aus untergeordneten Rängen kam, verdeutlichten der Heeresführung die Gefahren, die ein Erfolg der Rebellen für das Überleben der Institution mit sich gebracht hätte.

Beide demokratischen Regierungen sahen sich mit Streitkräften konfrontiert, die zwar fragmentiert, aber nicht ohne weiteres dazu bereit waren, auf ihre traditionellen Machtbereiche zu verzichten. Die vier bewaffneten Aufstände waren ein Reflex des Bemühens um eine stärkere Legitimation durch eine Infragestellung der politischen Macht. Sie spiegelten auch die vielfältigen internen Brüche innerhalb der Streitkräfte wider. Die Erhebungen wurden von Obersten angeführt, die größtenteils dem Heer angehörten und erkennbar regional verortet waren. Einerseits mag die Tatsache, dass es sich bei denjenigen, die die korporativen Forderungen auf gewaltsame Art und Weise vorbrachten, um Oberste handelte, eine gewisse beruhigende Wirkung haben, denn es ist schwer vorstellbar, dass 
ein von der mittleren Hierarchie getragener Staatsstreich Erfolg haben könnte; andererseits sorgt die Offensichtlichkeit des fehlenden institutionellen Gehorsams für Ungewissheit hinsichtlich der langfristigen Verpflichtung der Streitkräfte gegenüber den demokratischen Normen und Institutionen. Der neue Grundton, mit dem die Regierung Menem die Außenbeziehungen des Landes versehen sollte, ${ }^{5}$ entsprach auch einem veränderten internationalen Kontext und einer dringenden Notwendigkeit, sich in die globale Gemeinschaft einzugliedern. Die Regierung Alfonsín hatte dies nicht als prioritäre Aufgabe angesehen.

\section{Die Strategie der Regierung Menem gegenüber den Streitkräften}

Die Regierung Menem hoffte einerseits, dass die Streitkräfte zu einem Prozess der Selbstreinigung finden würden, indem sie diejenigen ausgrenzten, die die Befehlsstruktur missachtet hatten, und diejenigen bestraften, die einen Angriff auf die Bürgerrechte unternommen hatten. Die Ergebnisse des Selbstreinigungsprozesses waren minimal, aber sie führten erneut zu internen Auseinandersetzungen, denn die jungen Offiziere wollten nicht mit den Kosten von Operationen belastet werden, mit denen sie selbst nichts zu tun gehabt hatten. ${ }^{6}$ Aber vor die Alternative gestellt, die ehemaligen Vorgesetzten zu kritisieren und damit die internen Divergenzen in die Öffentlichkeit zu tragen oder das Image einer hierarchischen und strukturierten Organisation aufrechtzuerhalten, entschieden sie sich für die Verteidigung der Korporation.?

Zum anderen war die Regierung der Ansicht, dass die Streitkräfte sich modernisieren und reformieren müssten, um die politische Stabilität zu gewährleisten. Der neue politische Ansatz der Regierung Menem und die Kursänderungen im Bereich der Außenpolitik zwangen die Streitkräf-

5 Zur Außenpolitik siehe den Beitrag von Bodemer in diesem Band.

6 Dies wurde beispielsweise deutlich, als man Admiral Massera den Eintritt zum Círculo del Mar verweigerte, einer Organisation, der aktive und im Ruhestand befindliche Offiziere angehören (Clarín, 31.3.1998).

7 Diese korporative Logik erklärt die gemeinsame Reaktion der Streitkräfte angesichts der Weigerung des Kongresses, den Marineoffizieren Rolón und Pernía die anstehende Beförderung zu gewähren. Gleiches gilt für die Vertuschung der inneren Auseinandersetzungen innerhalb des Heeres, als man verhindern wollte, dass die öffentliche Meinung von den Vorbehalten vieler Offiziere angesichts der Reden von General Balza erführe, in denen dieser die Verantwortlichkeiten des Heeres im Rahmen des Schmutzigen Krieges anerkannt hatte. 
te dazu, sich Gedanken über ihre zukünftigen Aufgaben zu machen (Diamint 1995).

Obwohl sie explizit die demokratischen Regeln anerkannten, zeigten die Ausdrucksformen der Streitkräfte, dass sie nicht immer dazu bereit waren, sich der Regierung sowie den demokratischen Institutionen und Normen unterzuordnen. Dies wurde anlässlich der Vorstellung der „Strategischen Konzeption der Marine“ deutlich. Die entsprechende Direktive nützte eine vom Verteidigungsministerium nicht bedachte Gesetzeslücke und interpretierte diese im Sinne autonomer Spielräume ohne politische Kontrollen. Die Marine arbeitete einen strategischen Plan aus, in dem sie selbst ihre Aufgaben und die diesen zugrundeliegenden Kriterien definierte. Man hatte ein Treffen vorbereitet, an dem Regierungsfunktionäre, Parlamentarier und Medienvertreter teilnahmen und bei dem eine Publikation vorgestellt werden sollte, die diese Planungen öffentlich machte. Das Treffen wurde zwei Tage vor dem vorgesehenen Termin aufgrund einer Intervention durch Mitglieder der Verteidigungskommission des Kongresses abgesagt. Interessanterweise wurde der für die vorzeitige Veröffentlichung verantwortliche Konteradmiral José Roberto Fernández, dem man eine glänzende Karriere vorausgesagt hatte, in den vorzeitigen Ruhestand versetzt. Diese Entscheidung führte erneut zu einem Zerwürfnis innerhalb der Marine (Clarín, 30.10. 1993; Página 12, 31.10.1993).

Dieses Ereignis verdeutlichte die fehlende Kommunikation zwischen den Kommandanturen der Teilstreitkräfte sowie zwischen diesen und dem zuständigen Ministerium. Auch das Fehlen einer parlamentarischen Kontrolle wurde sichtbar. Ein Ausweg aus der verfahrenen Situation wurde durch eine Stärkung der institutionellen Struktur und Hierarchie gesucht. Dabei spielten sowohl Marinechef Kommandant Molina Pico als auch die Entscheidungen von Präsident Menem eine wichtige Rolle. ${ }^{8}$ Ein größerer Konflikt konnte vermieden werden, aber diese Mini-Krise brachte wenig transparente Verhaltensformen und überkreuzte Loyalitäten ans Licht. Zudem zeigte sich, dass das Verteidigungsministerium

8 Molina Pico betonte, dass Fernández ohne seine Erlaubnis zu einer Pressekonferenz in das Marinegebäude eingeladen habe. Angesichts der Art und Weise, wie innerhalb des Militärs mit Hierarchien umgegangen wird, ist dies jedoch unwahrscheinlich. Fernández bat um ein Ehrengericht, welches letztendlich zu der Ansicht kam, dass Molina Pico nichts vorzuwerfen sei; es verhängte eine leichte Sanktion gegen Konteradmiral Fernández und zeigte damit, dass die Institution über allen anderen Überlegungen steht (La Nación, 28.10. 1993; El Cronista Comercial, 2.11. 1993; El Cronista Comercial, 8.12.1993; El Litoral, 12.12.1993). 
nicht der wichtigste Ansprechpartner der Streitkräfte war. Die militärischen Führungsstäbe verließen sich nicht auf den formalen Dienstweg, sondern suchten den direkten Kontakt zum Präsidenten.

Während der Amtszeit von Präsident Menem fühlten sich die Streitkräfte dazu veranlasst, im Rahmen der von der Exekutive festgelegten Bedingungen zu verhandeln. Man erkannte, dass direkte Kontakte mit dem Präsidenten, Einflussversuche bei wichtigen außenpolitischen Themen oder die Nähe zur „Umgebung“ des Präsidenten bessere Ergebnisse versprachen als die Einhaltung des Dienstweges und der offizielle Dialog mit der Regierung. Beispielsweise war die UCR-Opposition im Kongress nicht dazu bereit, die Entsendung von Kriegsschiffen in den persischen Golf zu unterstützen, da man die dortigen Auseinandersetzungen als Teil der nordamerikanischen Ölinteressen und nicht als eine Bedrohung für den Weltfrieden betrachtete. Zudem fand die Entsendung der Schiffe an den Grenzen der Verfassungsmäßigkeit statt, insofern jede Kriegserklärung vom Kongress autorisiert werden muss. Für die Armee dagegen bot sich eine Möglichkeit, die Verbindungen zur Regierung zu verbessern und ihre Rolle als Profession zu stärken. Die Verantwortlichen zeigten keine Skrupel angesichts der Nichtbeachtung der verfassungsmäßigen Normen (Página 12, 22.8.1990 u. 24.8.1990; Clarín, 19.9.1990).

Menem wandte eine Strategie von Zuckerbrot und Peitsche an. Er war sich bewusst, dass die Regierbarkeit von seinem Geschick abhing, sich die traditionellen Korporationen der argentinischen Gesellschaft die Streitkräfte, die Kirche und die Gewerkschaften - nicht offen zu Feinden zu machen. Die Militärs ihrerseits nutzten die wenigen Möglichkeiten, in denen sie die privilegierten Akteure einer staatlichen Politik waren. So übten sie Druck auf den Verteidigungsminister aus, um diesen von der Unmöglichkeit der Einführung eines freiwilligen Militärdienstes zu überzeugen, wenn die Haushaltszuweisungen nicht erhöht würden. Ebenso erklärten sie, dass die menschlichen und materiellen Ressourcen für eine Teilnahme an internationalen Friedensmissionen nicht zur Verfügung stünden, wohlwissend, dass dies eine außenpolitische Priorität war.' Ein Offizier der Luftwaffe äußerte die Ansicht, dass der Regie-

9 „Wir werden ein Bataillon mit 300 Männern [nach Haití] entsenden, wie dies auch mit Zypern geschehen ist, wenn die finanziellen Voraussetzungen für eine solche Aufgabe gegeben sind. " Letztlich wurden 350 Mann entsandt, die mit Mitteln außerhalb des normalen Haushaltes finanziert wurden. Die Streitkräfte wussten sehr genau, dass für prioritäre Ziele immer die erforderlichen Mittel gefunden werden und dass dafür nicht auf die formale Struktur des Staatshaushaltes zurückgegriffen wird (Las Andes, 22.10.1994). 
rungsstil von Präsident Menem - das häufige Rekurrieren auf Dekrete und das caudillistische Gehabe - für die Streitkräfte ein Anreiz gewesen sei, ihre eigenen Machtsphären entgegen den vorgesehenen Regeln neu zu ordnen (Interview am 20.8.1998).

Die Strategie der Luftwaffe blieb stärker den Bedingungen des überkommenen Staatsmodells verhaftet, weshalb sie unter Menem die am wenigsten erfolgreiche der drei Teilstreitkräfte bei dem Bemühen war, ihre institutionellen Spielräume zu verteidigen. Hinsichtlich des Raketenprogramms Condor II nutzten die Verantwortlichen alle ihnen zur Verfügung stehenden Ressourcen, von der bürokratischen Legalität bis zum Lobbyismus und der Anmaßung. Ähnliche Verhaltensweisen zeigten sie, als es um den Kauf von Flugzeugen des Typs A4M ging. Zwischen dem Chef der Luftwaffe, Brigadegeneral Paulik, der von Rechts wegen dazu befugt ist, über die Ausrüstung seiner Streitkraft zu entscheiden, und den Verantwortlichen im Verteidigungs- sowie im Wirtschaftsministerium entwickelte sich ein Kompetenzkonflikt. Paulik war Verpflichtungen gegenüber der US-Luftwaffe eingegangen, während die Ministerien sich für ein Angebot des Unternehmens Lockheed entschieden. Auch in dieser Situation wandte sich der Luftwaffenchef unter Umgehung des Verteidigungsministeriums direkt an den Staatspräsidenten. Erneut wurde der Konflikt im Sinne der Luftwaffe und unter Nichtbeachtung der ministeriellen Kritiken gelöst. ${ }^{10}$

Die Haushalts- und Ausrüstungsfragen führten zu angespannten und schwierigen Verhandlungen. Zur gleichen Zeit, als die Sparmaßnahmen im Rahmen der Steuerreform beschlossen wurden, ließ man es zu, dass die Streitkräfte über Ressourcen außerhalb der parlamentarischen Kontrolle verfügten und so ihre eigene Beschaffungspolitik betreiben konnten. Beispielsweise kam aufgrund des Gerichtsfalles Yabrán die Beziehung zwischen der Luftwaffe und den Unternehmen Interbaires, Intercargo und Edcadassa ans Licht, die im Bereich Lagerhaltung, Lasten-

10 Um den Kauf der Flugzeuge des Typs Skywaks 4AM zu finanzieren, wurde ein zusätzlicher Haushaltsposten in Höhe von 315 Millionen Dollar nachträglich in das Budget für 1995 aufgenommen. Gleichzeitig wurde die Luftwaffenfabrik Area Material Córdoba an die nordamerikanische Firma Lockheed verkauft (La Nueva Provincia, 17.2.1994). Angeblich bewahrte nur Präsident Menem Luftwaffenchef Paulik vor der Versetzung in den Ruhestand, die bereits durch Verteidigungsminister Oscar Camilión ausgefertigt worden war (Clarin, 20.2.1994). 
transport und Duty Free Shops an Flughäfen tätig waren. ${ }^{11}$ Diese Geschäftsverbindung basierte auf Ressourcen, deren Verwendung weder durch die Legislative noch durch die Kontrollorgane der Exekutive überwacht wurde und die verschiedene Formen illegaler Transaktionen ermöglichten. Einige dieser Gelder stammten aus illegalen Waffenverkäufen: Während die Regierung im „Verzeichnis Konventioneller Waffen" (Registro de Armas Convencionales) den Ankauf von Ausrüstungsgütern nicht angab, wurden US-amerikanische Unterlagen bekannt, aus denen der Umfang der durch Argentinien getätigten Käufe hervorging. ${ }^{12}$ Daneben gab es andere Formen der Ressourcenbeschaffung, die zwar nicht illegal waren, aber ebenfalls nicht aus normalen Haushaltsmitteln herrührten. Dies galt beispielsweise für den Verkauf von Reparatur- und Bestandserhaltungsdienstleistungen, wodurch Kapitaleinkünfte mittels des privaten Verkaufs von Dienstleistungen erzielt wurden. ${ }^{13}$

Diese Entscheidungen der Streitkräfte waren weder transparent, noch wurden sie im Kongress debattiert oder basierten auf formellen Übereinkünften mit den verantwortlichen Ministerien. Die Möglichkeit zu entsprechenden Geschäften ergab sich nicht aufgrund von korporativem Druck, sondern als Ergebnis eines wechselseitigen Entgegenkommens zwischen Regierung und Militärs. Auf nicht-institutionelle Wege griffen somit sowohl die Streitkräfte als auch die politischen Entscheidungsträger zurück. Diese Strategien zeigen eine Präferenz zur Umgehung von Regulierungsmaßnahmen und institutionellen Verfahrensweisen. Das Handeln sowohl ziviler als auch militärischer Funktionsträger

11 Der Unternehmer Yabrán, welcher schließlich Selbstmord beging, war ein Paradebeispiel für die Korruption unter Beteiligung von Regierungen, Korporationen und illegitimen Wirtschaftsinteressen: Drogenhandel, Waffenschieberei, Bestechung und Veruntreuung (Clarin, 16.6.1998; La Nación, 3.7.1998). Auch die Beziehungen zwischen dem Bruder des Luftwaffenchefs Juliá und einem Waffenschieber standen damit in Verbindung (Clarin, 3.7.1998).

12 Die Tageszeitung La Nación veröffentlichte am 4. Juni 1998 folgende Meldung: „Der Tageszeitung La Nación liegen Informationen einer hochrangigen gerichtlichen Quelle vor, aus denen hervorgeht, dass die Situation des Heeres hinsichtlich der illegalen Versendung von Kanonen und Munition nach Kroatien sehr kompromittierend ist. " Siehe auch La Nación, 6. u. 23.6.1998. Die Explosion der Militärfabrik in Río Tercero war absichtlich herbeigeführt worden, um die illegale Verschickung von Rüstungsgütern zu vertuschen (La Nación, 3.11.1997). Siehe auch den Bericht von Douglas Farah, „A Tutor to Every Army in Latin América“, in: The Washington Post, 13.7.1998.

13 Die Marine führt beispielsweise die Wartung der Turbinen für die brasilianischen Streitkräfte durch. Durch ein günstiges Angebot konnte sie Großbritannien, das diese Aufgabe zuvor wahrnahm, aus dem Geschäft drängen. Auch die Ausbildung von Offizieren wird als Dienstleistung angeboten. 
zeichnet sich durch fehlende accountability und Praktiken am Rande der Legalität aus. Wir haben es hier auch mit einem Erbe des Autoritarismus zu tun, der parteilichen und verdeckten Handlungsweisen auf Kosten der Kontrollrechte zivilgesellschaftlicher Repräsentanten Schutz gewährte.

In den vergangen Jahren haben sich die Militärs zudem in gewandte und flexible Funktionäre verwandelt, die es verstehen, sich an sich ändernde interne und externe Rahmenbedingungen anzupassen. Sie können genauso als internationale Förderer des Friedens auftreten wie als Katastrophenhelfer. Gleichzeitig entstand ein neues Risiko für die Konsolidierung der Demokratie: zu den militärischen Missionen kamen polizeiliche Aufgaben hinzu, die die traditionellen Missionen der Landesverteidigung überlagerten, und dies in einer Gesellschaft, in der das Bedürfnis nach größeren Sicherheitsmaßnahmen stieg, weil die alltägliche Gewalt zunahm. ${ }^{14}$ Für die Militärs bot sich damit die Möglichkeit, neue Funktionen zu bekleiden, eine Quelle für Arbeitsplätze zu erschließen und ihre Rolle zu legitimieren. Die Dringlichkeit von Lösungen für die Gewährleistung der öffentlichen Ordnung verschaffte den Militärs gröBere Verhandlungsspielräume mit den zivilen Entscheidungsträgern.

\section{Militärpolitische Entscheidungsprozesse}

Eines der zentralen Themen, das wiederholt Anlass zu Konflikten zwischen Militärs und Regierung gab, war der Entscheidungsprozess in verteidigungspolitischen Fragen und hinsichtlich der Umstrukturierung des Militärhaushaltes. Die Neuordnung der öffentlichen Finanzen war der Ausgangspunkt für eine Kürzung der Verteidigungsausgaben und damit auch für die Definition des neuen Stellenwertes der Streitkräfte innerhalb der staatlichen Institutionen. Aus einer institutionellen Perspektive betrachtet, musste sich die Überwindung der autoritären Militärtradition in einer eindeutigen Unterordnung der Streitkräfte unter das für die Verteidigungspolitik verantwortliche Ministerium niederschlagen. Seit der Rückkehr zur Demokratie wurde das Verteidigungsministerium von Zivilisten geleitet, aber in den Führungsetagen fanden sich in der Regel zahlreiche Offiziere im Ruhestand. Die Loyalität dieser Funktionäre galt in erster Linie ihrer jeweiligen Waffengattung, in zweiter Linie den Streit-

14 Im Jahr 1996 gab es 171.000 Polizisten und 32.000 Sicherheitskräfte der Präfekturen und Gendarmerien, die dem Innenministerium unterstehen; dem standen 76.000 Militärangehörige gegenüber, die dem Verteidigungsministerium unterstellt sind $(E l$ Porteño 1, September 1996: 11). 
kräften insgesamt und erst an dritter Stelle der zivilen Regierung. ${ }^{15}$ Die Verantwortung dafür, dass sich das Ministerium nicht an die Notwendigkeiten der Demokratie anpasste und den Militärs weiterhin große Spielräume bot, lag allerdings bei den zivilen Entscheidungsträgern. Ähnlich sah es mit den Zuwendungen im Rahmen des Verteidigungshaushaltes aus. Das Finanzverwaltungsgesetz sieht vor, dass die Ressourcen auf Anforderung des Verteidigungsministers durch den Finanzminister zugewiesen werden, wobei die Zustimmung des Bundesparlamentes notwendig ist. In der Realität erfolgen die Zuwendungen auf der Grundlage überkommener Kriterien und von den Kommandanten der Teilstreitkräfte vorgelegter Anträge. Die tatsächlichen Möglichkeiten des Verteidigungsministers, über die Verwendung der Gelder zu verfügen, sind minimal. Wenn es hoch kommt, kann er die von den Teilstreitkräften präsentierten Anforderungskataloge an der einen oder anderen Stelle kürzen, je nachdem, wie hoch die Zuwendungen durch das Finanzministerium sind (Diamint 1994). Infolgedessen bestimmt nicht der zuständige Minister in erster Linie über die Ausgaben der Teilstreitkräfte und die damit zu finanzierenden Aktivitäten, sondern das Militär selbst.

Doch auch wenn es den Streitkräften nach der Rückkehr zur Demokratie gelungen ist, weitgehend selbst über die Verwendung ihrer Ressourcen zu entscheiden, so sahen sie sich doch mit rigiden Positionen hinsichtlich des Anteils des Verteidigungshaushaltes an den Gesamtausgaben des Staates konfrontiert. Die Streitkräfte treten mit anderen staatlichen Institutionen, die sich in der gleichen Situation befinden, in einen Wettbewerb um die Verteilung des Haushalts. Auch zwischen den Waffengattungen gibt es ein Tauziehen um die finanziellen Zuwendungen. Jede Streitkraft muss sich Strategien überlegen, um ihre eigenen finanziellen Bedürfnisse so effizient wie möglich darzustellen. Hochrangige Offiziere (insbesondere der Marine) belegten sogar Kurse an prestige-

15 Noch 1996 erhoben einige der teilnehmenden Delegationen aus Anlass der zweiten Konferenz der Verteidigungsminister Amerikas Einwände dagegen, dass eine der Arbeitsgruppen „Die Rolle der Streitkräfte in der Demokratie“ betitelt werden sollte. Sie forderten, dies durch „Die Rolle Der Streitkräfte im 21. Jahrhundert“ zu ersetzen (Information des Ministerio de Relaciones Exteriores, Comercio Internacionaly Culto, 12. Juni 1996). Ein anderer Fall war der eines ehemaligen Generals, der die Abteilung Militärpolitik des Verteidigungsministeriums leitete. Anlässlich eines bilateralen Treffens mit Brasilien legte er ein informelles Papier (non-paper) vor, in dem ein Schema für gemeinsame Militärinterventionen in Fragen des Drogenhandels vorgeschlagen wurde. Dies widersprach den rechtlichen Bestimmungen und führte zum erzwungenen Rücktritt des Abteilungsleiters. 
trächtigen Bildungseinrichtungen wie dem Massachussets Institute of Technology oder der Naval Postgraduate School in Monterrey, um sich selbst in die Lage zu versetzen, ihre Haushaltsentwürfe gemäß den komplizierten Mechanismen des neuen Haushaltsrechts ausarbeiten zu können.

Parallel zu dieser zurückhaltenden Akzeptanz der Spielregeln bemühten sich die Streitkräfte darum, jede Verantwortung für eine defizitäre Verwendung der erhaltenen Ressourcen von sich zu weisen. In einem Schreiben des Heeres an das Verteidigungsministeriums war zu lesen: „Das Funktionieren des Heeres darf nicht durch Sparzwänge und sinkende Mittelzuweisungen, die oftmals ohne vorhergehende Konsultation stattfinden und zu schwierigen Situationen führen, in Mitleidenschaft gezogen werden." (La Nación, 24.11.1994) In diesem Schreiben wies das Heer jede Verantwortung für eine Nichterfüllung seiner öffentlichen Aufgaben in dem Maße zurück, wie ihm nicht die notwendigen Ressourcen für die Realisierung seiner in der Verfassung festgelegten Funktionen zugebilligt würden. Dementsprechend könne die Gesellschaft dem Heer weder Nachlässigkeit vorwerfen, noch es für die fehlende Sorgfalt bei der Instandhaltung seiner Ausrüstung verantwortlich machen. Die Sparpolitik wird als den nationalen Interessen widersprechend dargestellt, obwohl es vor allem die Interessen der Streitkräfte als eines privilegierten Akteurs sind, die dadurch Einschränkungen erfahren.

\section{Die zivil-militärischen Beziehungen}

Die meisten Studien über die Militärkultur verweisen nachdrücklich auf die Prägung der Streitkräfte durch die verschiedenen Ausbildungseinrichtungen im Verlauf der Offiziersausbildung. Infolgedessen legte man während der Amtszeit von Präsident Alfonsín besonderen Wert auf die Vermittlung und Internalisierung demokratischer Werte und hoffte darauf, dass dadurch die autoritären Tendenzen aus dem kulturellen Horizont des Militärs verschwinden würden. Das Verteidigungsministerium bemühte sich insbesondere um die Erarbeitung neuer Ausbildungsprogramme, deren Inhalte demokratischen Regeln und Konzeptionen entsprechen und Respekt gegenüber den Bürgern vermitteln sollten. Aber die Militärinstitutionen lehnten eine Einmischung von außen ab und neutralisierten die neuen Inhalte dadurch, dass sie an ihren Schulen weiterhin die alten Professoren beschäftigten. Dementsprechend waren die Erfolge in diesem Bereich nur begrenzt.

Ähnliche Versuche wurden, allerdings weniger systematisch, während der Präsidentschaft von Carlos Menem initiiert. Verteidigungs- 
minister Camilión wollte die Nationale Verteidigungsakademie (Escuela Nacional de Defensa; END) ,zivilisieren“ und griff dazu auf ähnlich geartete Ansätze zurück wie die UCR-Regierung. Zum Direktor der END ernannte er einen Diplomaten aus seinem persönlichen Freundeskreis, der die nicht gerade als Hort der Demokratie bekannte Einrichtung zur Keimzelle einer neuen Generation von Funktionären machen sollte, die eine zentrale Rolle im Rahmen der zukünftigen Verteidigungspolitik spielen würden. Als Modell diente die Verteidigungsuniversität der Vereinigten Staaten von Amerika, aber das Vorhaben scheiterte an der Weigerung des Heeres, der zivilen Verwaltung eine Institution zu ,übergeben", die als die eigene betrachtet wurde. ${ }^{16}$

Auf der Suche nach mehr gesellschaftlicher Anerkennung präsentierten die Streitkräfte ihrerseits mehrere Vorschläge, die zu einer Annäherung zwischen Zivilisten und Militärs führen sollten. Das Heer organisierte Kurse für Journalisten, ${ }^{17}$ bot Filmvorführungen zu militärischen Themen an, bereitete eine Modenschau im traditionsreichen Regiment von Patricios vor und lud Familien mit Kindern zu einem Freizeitvergnügen ein, bei dem im Regiment von Palermo Kriegsspiele simuliert wurden. Zudem wurden zwischen dem Sekretariat für Menschenrechte und der Nationalen Gendarmerie Abkommen über Erziehungsmaßnahmen im Bereich Menschenrechte unterzeichnet (La Nación, 19.4.1998). Die Marine schickte einen Flottenverband in den Hafen von Buenos Aires und regte Kinder dazu an, Soldaten zu spielen.

Die entscheidende Frage für die Beziehungen zwischen Militärs und Gesellschaft bleibt jedoch die Behandlung des Themas Menschenrechte. Die Reden, in denen General Balza die institutionelle Verantwortung des Heeres für Folter und Repression während der Militärdiktatur anerkannte, bedeuteten einen spektakulären Wandel im Vergleich zu den traditionellen Positionen der Streitkräfte. Über die Gehorsamspflicht der Soldaten äußerte er sich in einer Rede anlässlich der Abschlusszeremonie des Jahrgangs 1998 in der nationalen Kadettenanstalt folgendermaßen:

16 Die DNG ist funktional und haushaltsmäßig dem Verteidigungsministerium unterstellt, aber die Abschlüsse werden durch die Provinzuniversität des Heeres (Universidad Provincial del Ejército) vergeben.

17 Im Rahmen seines Abschlussvortrages sagte General Balza: „Die alte Vorstellung, nach der die Militärs eingeschlossen in ihrer Festung lebten, hat an Gültigkeit verloren, denn jetzt stehen die Kasernentore für jedwede journalistische Recherche offen." (La Nación, 3.12.1994). 
Gehorsam schuldet man nur legitimen Befehlen, die von legitimen Autoritäten gegeben werden. Erinnern Sie sich daran, dass man Ihnen das Recht gibt, Waffen zu tragen [...], aber dieses Recht gründet auf der Verfassungsnorm, welche die Rolle der Streitkräfte als Zwangsinstrument legitimiert. Nur wer an die Verfassung und an die Gesetze glaubt, kann verstehen, dass die legitime Regierung aus dem souveränen Willen des Volkes entspringt, welches seinen Repräsentanten sein eigenes Recht überträgt. Denken Sie deshalb daran, dass diese Waffen nur dann benutzt werden dürfen, wenn die verfassungsmäßige Macht es so verfügt. (zitiert nach La Prensa, 13.12.1994).

Die Erklärungen des Heereskommandanten stießen auf die Zustimmung der Zivilgesellschaft und auf deutliche Ablehnung bei den Offizieren im Ruhestand. General Balza ging es um eine Versöhnung zwischen dem Heer und der Gesellschaft. Gleichzeitig sollte die Einheit des Heeres wiederhergestellt werden, aber es gelang ihm nicht, die Brüche zwischen den Offizieren und ihren Vorgesetzten zu überwinden (Clarin, 5.5.1995).

Sowohl Präsident Menem als auch General Balza bemühten sich um eine Erneuerung der Beziehungen zwischen Streitkräften und Gesellschaft. Aber dem Heereskommandanten ging es auch darum, das Unbehagen einiger seiner Untergebenen und vieler Offiziere im Ruhestand zu minimieren, denn sein vorrangigstes Interesse galt der Erneuerung und dem Erhalt der Einheit seiner Teilstreitkraft. Gegenüber seinen Gegnern innerhalb des Heeres reagierte er mit großer Entschiedenheit. Er zeigte sich davon überzeugt, dass die öffentliche Reue das Heer erneuern und stärken und zur Neudefinition seiner Rolle im politischen Leben beitragen werde. Diese Anstrengungen wurden jedoch durch zwei andere Ereignisse in Mitleidenschaft gezogen, durch die die guten Absichten und die Glaubwürdigkeit des Heeres in Frage gestellt wurden. Es handelte sich um den Tod eines Soldaten in Folge von Misshandlungen durch seine Vorgesetzten sowie um den Verkauf von Waffen nach Kroatien und Ecuador. Beide Fälle ließen Zweifel hinsichtlich der moralischen Integrität des Heereskommandanten aufkommen.

Während Balza öffentlich Reue zeigte, suchte die Marine die Schuld bei anderen und räumte der Verteidigung ihrer Mitglieder Priorität ein. Dies war schon im Fall der Offiziere Rolón und Pernía so gewesen, als deren Beförderung von Menschenrechtsorganisationen und Abgeordneten der Radikalen Partei in Frage gestellt worden war. Der Oberkommandierende der Marine, Admiral Molina Pico, stellte sich hinter die Forderungen seiner Streitkraft und brachte die Angelegenheit an höchster Stelle vor. Auch wenn er damit keinen Erfolg hatte, so sorgte sein 
Verhalten doch dafür, dass er von Kritik aus den eigenen Reihen verschont blieb. Die Selbstkritik von Molina Pico, die dieser im Namen des Admiralsrats vortrug und nicht in persönlicher Form wie im Falle von General Balza, zeichnete sich durch einen stärker rechtfertigenden Charakter aus. Von ihm waren Sätze zu hören wie: „Das Vorgehen der Streitkräfte darf nicht so analysiert werden, als ob sie die einzigen Beteiligten an der Angelegenheit gewesen wären." (Clarin, 4.5.1995) oder „Die Nation wurde angegriffen und hat sich verteidigt." (La Nueva Provincia, 4.5.1995). Die halbherzige Selbstkritik der Marine stand in besonderem Kontrast zu der Tatsache, dass die Vorwürfe des Kapitäns Scilingo, der die Krise ins Rollen gebracht hatte, insbesondere die Marine kompromittierten. Die mit dieser Streitkraft in Verbindung stehenden Fälle hatten den stärksten Nachhall in der Öffentlichkeit gefunden.

Was die Luftwaffe angeht, so verband General Paulik die Auseinandersetzung mit den Verbrechen während des „Schmutzigen Krieges“ mit Forderungen an die Regierung, für ein akzeptables Funktions- und Ausrüstungsniveau seiner Streitkraft zu sorgen. Seine Selbstkritik ging nicht auf eine interne, von den Waffenkameraden geteilte Revision zurück, sondern auf eine Aufforderung durch die Regierung.

Kurze Zeit nach diesen Reden nahm die Gesellschaft mit Überraschung die Erklärungen des ehemaligen Kapitäns Alfredo Astiz zur Kenntnis, der ausführlich die während der Diktatur begangenen Gräueltaten schilderte und diese als Teil einer institutionellen Politik der Marine bezeichnete (Cerruti 1998; Clarin, 16.4.1998). Diese Tatsache, genauso wie die spätere Anklage gegen General Videla wegen Kindesraubes, legt die nach wie vor tiefgreifenden Brüche innerhalb der Gesellschaft offen. General Balza selbst erkannte dies in einer Rede an:

Das Heer ist sich seiner institutionellen Verantwortung bewusst, es bemüht sich darum, einen schmerzhaften, nicht geführten Dialog in Gang zu bringen über eine Vergangenheit, die nach wie vor eine offene Wunde im kollektiven Bewusstsein der Argentinier darstellt (12.2.1998).

Die Begrenztheit der Reuegefühlte und die Fortexistenz autoritärer Konzeptionen zeigte sich im Fall Carrasco. Der Soldat Carrasco, ein Rekrut, der einer Familie aus dem Süden des Landes entstammte, starb infolge brutaler Misshandlungen, denen er wegen seiner religiösen Überzeugungen ausgesetzt war. Der Leichnam wurde erst Tage nach einer durch seine Familie aufgegebenen Vermisstenanzeige gefunden. Es kam zu einem Prozess, in dessen Verlauf eine ganze Kette von Vorgängen aufgedeckt wurde, durch die die Ehrenhaftigkeit und die ethischen Überzeugungen 
der Heeresangehörigen erneut in Zweifel gezogen wurden. General Balza antwortete auf den Vorfall im Namen des Heeres: „Es handelt sich um ein abscheuliches Verbrechen [...] Ich empfinde große Scham und ich werde sie noch viel stärker empfinden, wenn sich Beweise dafür finden was ich glaube - dass Angehörige der Streitkräfte in den Fall Carrasco verwickelt sind." (Clarin, 19.9.1995). Trotzdem hatte die Gesellschaft das Gefühl, dass Willkürakte und Verbrechen vertuscht werden sollten, zumal sich schnell zeigte, dass es sich nicht um einen Einzelfall handelte. Die Auswirkungen waren derart negativ, dass Präsident Menem daraufhin beschloss, per Dekret die Wehrdienstpflicht abzuschaffen. Dies entsprach einer Forderung der Bevölkerung, deren Umsetzung über die institutionellen Kanäle nicht möglich gewesen war. Sowohl die Parteilichkeit, die im Verlauf der Verfahren vor der lokalen und der Militärjustiz deutlich wurde, als auch die Art und Weise, wie über die Schaffung eines Freiwilligen Militärdienstes entschieden wurde, verweisen auf die starke Personalisierung der argentinischen Politik.

Fehlende Transparenz und zweifelhafte Ethik kompromittierten das Heer auch im Falle der Waffenverkäufe nach Kroatien und Ecuador, während beide Länder sich im Krieg befanden. Die juristische Aufarbeitung dieser Angelegenheit ist noch nicht abgeschlossen, aber zweifellos handelt es sich um Beispiele für ungesetzliche Praktiken der Streitkräfte, für Korruption und für Klientelismus. Sowohl der Tod des Soldaten Carrasco als auch die Waffenverkäufe sind Belege für autoritäre Verhaltensweisen der Streitkräfte, die auf Praktiken zurückgehen, wie sie während der Militärdiktatur üblich waren. Wie in den Jahren der Diktatur spüren die Militärs auch angesichts ziviler Regierungen keine Verpflichtung, der Gesellschaft Rechenschaft über ihre Handlungen abzulegen. Verschlimmert wird das Ganze noch dadurch, dass die Korruptionsfälle von den zivilen Regenten toleriert werden, die sich damit dem Hochmut der Macht anschließen.

\section{Die Verteidigungspolitik}

Als Ergebnis der Demokratisierungswelle und des Endes der Spannungen im Rahmen des Kalten Krieges verflüchtigten sich die wichtigsten Konflikthypothesen, auf denen die argentinischen Streitkräfte ihr Selbstverständnis aufgebaut hatten. Eine Reihe von Konflikten, die in früheren Zeiten fast zum Ausbruch kriegerischer Auseinandersetzungen geführt hätten, konnten auf friedliche Art und Weise beigelegt werden. Dazu gehörten die Abkommen über die Beilegung der Grenzstreitigkeiten zwi- 
schen Chile und Argentinien und das gemeinsame argentinisch-brasilianische Nuklearprogramm.

Gleichzeitig kam es zu einer Ausweitung gemeinsamer militärischer Unternehmungen. Dies gilt beispielsweise für die Einsätze im Rahmen von UNITAS, an der auch Militärs aus den USA, Brasilien und Uruguay beteiligt waren und die zu einer Stärkung des gegenseitigen Vertrauens innerhalb der Region beitrugen. Die gemeinsamen Flottenmanöver Argentiniens und Chiles im Südatlantik, die zunächst auf beiden Seiten auf Widerstand gestoßen waren, entwickelten sich zu einem historischen Schritt für die bilateralen Militärbeziehungen. Auch das gemeinsame Auftreten argentinischer und chilenischer Militärs auf Zypern ist in diesem Rahmen zu sehen. Zweifellos reduzieren diese neuen Beziehungsmuster zwischen den Streitkräften der Region die Gefahr bewaffneter Auseinandersetzungen. Auch die Schaffung des Mercosur mit den Mitgliedern Argentinien, Brasilien, Uruguay und Paraguay sowie Chile und Bolivien als assoziierten Mitgliedern trug dazu bei, aus alten Feinden Partner zu machen.

Dadurch konnten die Beziehungen zu den Vereinigten Staaten gestärkt und die Einbindung des Landes in den internationalen Kontext verbessert werden. Viele Experten gehen auch davon aus, dass die Durchführung begrenzter militärischer Einsätze im Ausland zu einer Stärkung der zivilen Kontrolle über die Streitkräfte beiträgt (PionBerlin/Arceneaux 2000). Sie weisen aber auch darauf hin, dass dies nicht automatisch geschieht. Damit derartige Erfolge tatsächlich erzielt werden, müssen die entsprechenden Entscheidungen durch die zivilen Amtsträger getroffen und von den Streitkräften akzeptiert werden.

Die unter Menem erfolgten Beteiligungen an internationalen Friedensmissionen waren derjenige Bereich, in dem die stärkste Kontrolle über die Streitkräfte ausgeübt werden konnte und die besten Erfahrungen hinsichtlich der Zusammenarbeit zwischen verschiedenen Verwaltungsbehörden gemacht wurden. Die Entscheidungen über die Beteiligung an Auslandseinsätzen wurden auf ministerieller Ebene getroffen und mit den Streitkräften abgestimmt. Allerdings war das Außenministerium mit den entsprechenden Verhandlungen beauftragt, was damit zusammenhängt, dass dieses Ministerium auch für die Beziehungen mit den Vereinten Nationen zuständig ist. Es deutet aber auch darauf hin, dass das Außenministerium es besser als das Verteidigungsressort verstand, mit den Militärs umzugehen.

Seit 1990 verlagerte sich das Gravitationszentrum der Entscheidungen über die Sicherheitspolitik ins Außenministerium, dessen zentrale 
Zielsetzungen im militärischen Bereich lauteten: Nichtverbreitung von Kernwaffen, friedliche Lösung von Konflikten und Kooperation im Bereich der globalen Sicherheit. Zur Verwirklichung dieser Ziele diente sowohl die Beteiligung an den Friedensmissionen der Vereinten Nationen als auch die Mitarbeit im Rahmen der Rüstungskontrolle. Diese Politik war erfolgreich und führte zu einer Annäherung Argentiniens an die westlichen Länder, sie stieß aber auch auf nicht zu übersehende interne Widerstände.

Das Verteidigungsministerium ergänzte die Aufgaben des Außenministeriums, es ließ dabei allerdings klar durchblicken, dass nicht die Bevölkerung, sondern das Militär seine wichtigste Klientel sei. Obwohl es die Direktiven der Exekutive unterstützte, war es wesentlich weniger effektiv bei der Definition seiner Politik und bei der Verdeutlichung seiner Ziele. Die mangelhafte Koordination zwischen den Funktionären des Ministeriums und den Streitkräften lähmte manche Initiative und führte zu Spannungen innerhalb des Kabinetts. Das Ministerium bemühte sich kaum darum, eine Gruppe von Fachleuten auszubilden und dadurch eine Aufwertung der Verteidigungsaufgabe im Rahmen der allgemeinen politischen Richtlinien der Regierung durchzusetzen. Im neuen Gesetz zur Neuordnung der Streitkräfte wurde das Thema nicht einmal erwähnt. Zwar schreibt das Gesetz vor, dass das Verteidigungsministerium über Aufrüstung und Logistik bestimmen soll, entsprechende Maßnahmen wurden jedoch nicht getroffen.

Am 27. Juni 1995 wurde das Centro Argentino de Entrenamiento Conjunto para Operaciones de Paz (CAECOPaz) geschaffen, in dem die argentinischen Militärs auf ihren Einsatz im Rahmen von Friedensmissionen der UNO vorbereitet werden. Auch Soldaten aus anderen lateinamerikanischen Ländern kommen hierher, weshalb das Zentrum auch als Instrument zur Verbesserung der Beziehungen mit den Streitkräften der Nachbarländer dient.

\section{Abschließende Überlegungen}

Die Umgehung vorgeschriebener rechtlicher Kanäle durch die Streitkräfte, Einflussversuche mittels direkter Kontakte zum Präsidenten und der Einsatz halb-legaler Ressourcen zur Durchsetzung militärischer Interessen - derartige Restbestände militärischer Selbstherrlichkeit untergraben die demokratischen Institutionen. Eine solche Vorgehensweise unterscheidet sich allerdings nicht allzu sehr von der Logik der Machtakkumulation, wie sie die zivilen politischen Kräfte betreiben. Im Ergebnis neh- 
men die Auseinandersetzungen zwischen verschiedenen Fraktionen innerhalb des Staates zu, wobei es nicht in erster Linie um institutionelle Errungenschaften, sondern um persönliche Vorteile geht. Nimmt man zu diesem Panorama Gefahren bzw. Herausforderungen wie den Drogenhandel, die Umweltverschmutzung, den Terrorismus und die organisierte Kriminalität und eine zukünftige Beteiligung der Militärs an Aufgaben im Bereich der inneren Sicherheit hinzu, so ist unbedingt eine Stärkung der parlamentarischen Kontrollmechanismen notwendig, wenn es nicht zu einer weiteren Verschlechterung der Beziehungen zwischen Zivilgesellschaft und Streitkräften kommen soll.

Die bislang auf diese Herausforderungen gegebene Antwort, nämlich der Versuch einer stärkeren (staatsbürgerlichen) Erziehung der Militärs, ist in diesem Zusammenhang zwar ein wichtiger, aber lediglich ein sekundärer Aspekt, der die zentrale Problematik ausblendet. Dies zeigt sich auch an den bislang nicht besonders erfolgreichen Bemühungen des $\mathrm{Co}$ mando Sur, zur staatsbürgerlichen Erziehung der lateinamerikanischen Militärs und damit zur Demokratisierung der Streitkräfte innerhalb der Region beizutragen. Die Militärs beten die Lektionen über Menschenrechte nach, aber wenn sie dann nicht mehr im Klassenraum sitzen, sondern konkrete Aufgaben realisieren, greifen sie erneut auf die traditionellen Repressions- und Zwangsmaßnahmen zurück. Der Wandel darf sich daher nicht auf erzieherische Maßnahmen beschränken, sondern vor allem müssen Machtressourcen entwickelt werden, um die zivile Suprematie über die Streitkräfte effektiv zur Geltung zu bringen.

Nun wäre es ebenfalls falsch zu glauben, dass die Streitkräfte nach wie vor voll und ganz ihren überkommenen Konzeptionen treu geblieben sind. Ihre wichtigste Forderung lautet heute, als legitime Akteure im Rahmen der Entscheidungsprozesse über Sicherheitsfragen anerkannt zu werden. Die Militärs haben die Erfahrung gemacht, dass die einzige Möglichkeit zur Bewahrung ihrer Institution in einer Welt, in der der militärische Faktor an Gewicht verliert und die politische und ökonomische Effizienz des Staates die wichtigste Machtquelle ist, darin besteht, über einen modernen Staat zu verfügen, der ausreichende Ressourcen für die Unterhaltung der Streitkräfte bereitstellt. Carlos Acuña und Catalina Smulovitz (1996: 192) betonen, dass ,die Unterordnung der Streitkräfte unter die konstitutionelle Macht nicht aufgrund von entstehenden demokratischen Werten geschieht, sondern durch die Erkenntnis, dass bei Überschreitung gewisser Grenzen das Überleben des Akteurs an sich in Gefahr gerät.“ Die durch die Anpassungspolitiken, die Öffnung der Märkte und die politische Stabilität etablierten Rahmenbedingungen las- 
sen den Streitkräften keinen Spielraum, um ihre Forderungen losgelöst von dem neuen Staatsmodell zu entwickeln.

Es existiert ein Spannungsverhältnis zwischen der Rechtfertigung einer Zwangsgewalt, die den Streitkräften von der Gesellschaft zu ihrem eigenen Schutz zuerkannt wird, einerseits, und dem Widerstreben der Militärs zu gehorchen, das auf der gleichen Gewaltbefugnis basiert, andererseits (Zagorski 1992; Feaver 1996). Auch wenn die Streitkräfte nicht daran denken, einen Staatsstreich durchzuführen, können ihre politische Autonomie und das Fehlen gesetzlicher Kontrollen zu einer systematischen Einschränkung der zivilen Kontrolle führen. Notwendig ist die vollständige Etablierung ziviler Suprematie, d.h. die Fähigkeit der demokratisch gewählten Regierung, über die nationale Verteidigung zu bestimmen und die Durchführung der von ihr angeordneten Maßnahmen zu überwachen (Aguero 1995: 41-52). Nur so kann die Unsicherheit hinsichtlich der langfristigen Treue der Streitkräfte gegenüber den zivilen Herrschaftsträgern beseitigt werden.

In den gegenwärtigen marktwirtschaftlich orientierten Demokratien ist es wenig wahrscheinlich, dass die Ziele der Regierung mit den Interessen der Streitkräfte übereinstimmen. Beispielsweise nimmt ein Teil der Militärs das Voranschreiten der regionalen Integrations- und Kooperationsprozesse als Gefahr für die nationalen Interessen wahr. Auch in Argentinien hat ein Sektor der Streitkräfte sich um eine Annäherung an Politiker bemüht, die sich für eine Rückkehr zu einer stärker nationalistisch orientierten Politik aussprechen (Rico 1997). Solange die Militärs die Verteidigung der eigenen Korporation als ihr wichtigstes Ziel betrachten, wird es schwierig sein, zu einem dauerhaften Konsens hinsichtlich gesellschaftlicher Normen und Institutionen zu gelangen. Mehr noch, solange die Subordination der Streitkräfte unter die zivilen Machthaber nicht auf der Grundlage legal-rationaler Kriterien geschieht, sondern informell, in Funktion von Machtpfründen, büßen die Militärs Spielräume für institutionalisierte Verhandlungen ein und verfügen über weniger Mittel, um ihre gemeinsamen oder je nach Waffengattung spezifischen Interessen durchzusetzen. Aber auch wenn die institutionellen Forderungen geschwächt werden, erlauben es ihnen die persönlich geführten Verhandlungen über den Austausch von Gefälligkeiten, ihre korporativen Vorrechte innerhalb des demokratischen Rahmens zu bewahren.

Die Theorie geht davon aus, dass ein durch Zusammenbruch der Militärherrschaft erfolgter Übergang zur Demokratie die Streitkräfte als politischen Akteur eliminiert. Aber wie wir gesehen haben, verschwanden die Streitkräfte in Argentinien weder von der politischen Bühne, 
noch fand ein grundlegender Wandel ihrer korporativen Kultur statt. Es stellt sich die Frage, inwiefern die Fortexistenz autoritärer Legate eine Bedrohung für die Stabilität der demokratischen Institutionen darstellt. Worin besteht das Risiko? Die Militärs passten sich an die Strukturreformen und die Anpassungspolitiken auf patrimoniale Art und Weise an und konnten ihre Machtquellen erhalten. An die Demokratie passten sie sich in korporativer Manier an und bemühten sich darum, ihre Privilegien zu bewahren. Sie verfügen heute über weniger Ressourcen als in früheren Zeiten, um die zivile Macht in Frage zu stellen, aber es ist nicht gelungen, genügend gesellschaftliche Kapazitäten zu entwickeln, die zu einer institutionellen Stärkung der Demokratie und zum Abbau spezifischer Vorrechte der Streitkräfte beitragen könnten.

Die neoliberalen Anpassungspolitiken und Staatsreformen haben zu einer stärkeren politischen Kontrolle der Streitkräfte beigetragen. Die neue Staatsstruktur sorgt dafür, dass die Militärs nur noch eine Randposition innerhalb des Herrschaftsmodells einnehmen. Infolge der Sparpolitik sehen sich die Streitkräfte zunehmend in einer Verhandlungsposition, die sich nicht sonderlich von der anderer gesellschaftlicher Akteure unterscheidet. Aber auch wenn in Argentinien die Macht der Streitkräfte zur Durchführung eines Staatsstreiches gebrochen werden konnte, so gilt dies nicht für autoritäre Verhaltensweisen und Legate, die das politische Regime abqualifizieren und Defizite im Hinblick auf das Funktionieren der Demokratie nach sich ziehen. Letzten Endes hat sich die Demokratie konsolidiert, ohne eine zufriedenstellende Lösung für die Frage der zivilmilitärischen Beziehungen zu finden.

\section{Literaturverzeichnis}

Acuña, Carlos/Smulovitz, Catalina (1996): „Ajustando las Fuerzas Armadas a la democracia: Las Fuerzas Armadas como actor político en la experiencia del cono sur" in: Agora. Cuaderno de estudios politicos 5, 97-133.

Acuña, Carlos (Hrsg.) (1995): La nueva Matriz Politica Argentina, Buenos Aires: Nueva Visión.

Agüero, Felipe (1995): Militares, Civiles y Democracia. La España postfranquista en perspectiva comparada, Madrid: Alianza Editorial.

Agüero, Felipe (1992): „The Military and the Limits to Democratization“, in: Mainwaring, Scott/O’Donnell, Guillermo/Valenzuela, J. Samuel (Hrsg.): Issues in Democratic Consolidation: The New South American Democracies in Comparative Perspective, Notre Dame: University of Notre Dame Press, 153-198. 
Barkey, Henri J. (1990): „Why Military Regimes Fail: The perils of Transition“, in: Armed Forces and Society, Winter.

Cerruti, Gabriela (1998): „El asesino está entre nosotros“, in: Tres Puntos 28, 14.1.

Diamint, Rut (1994): „Gasto militar y ajuste económico en Argentina“, in: Rojas Aravena, Francisco (Hrsg.): Gasto Militar en América Latina. Procesos de decisión y actores claves, Santiago de Chile: FLACSO/CINDE, 139-181.

Diamint, Rut (1995): „Política exterior, seguridad regional y medidas de confianza mutua", in: Cáceres, Gustavo/Sheetz, Thomas (Hrsg.): Defensa no provocativa. Una propuesta de reforma militar para la Argentina, Buenos Aires: Editora Buenos Aires, 325-363.

Diamint, Rut (1997) (Hrsg.): „La toma de decision en asuntos de seguridad“, Buenos Aires: Universidad Torcuato Di Tella (Working Paper No. 39).

Diamint, Rut (1997a): El gobierno norteamericano ante el caso del Cóndor II. Sistema burocrático y toma de decisiones, Washington: Woodrow Wilson International Center for Scholars (Working Paper 224).

Diamint, Rut (Hrsg.) (1999): Control civil y fuerzas armadas en las nuevas democracias latinoamericanas, Buenos Aires: Editorial GEL.

Estévez, Eduardo (1990): „Aspectos salientes de la relación gobierno-fuerzas armadas 1983-1989“, in: Hacia una nueva relación, el papel de las Fuerzas Armadas en un gobierno democrático, Buenos Aires: National Democratic Institute for International Affairs.

Feaver, Peter D. (1996): „The Civil-Military Problematique: Huntington, Janowitz, and the Question of Civilian Control", in: Armed Forces and Society, 23 (2), 149-178.

Huntington, Samuel (1957): The Soldier and the State: The Theory and Politics of Civil-Military Relations, Cambridge: Harvard University Press.

Pion-Berlin, David/Arceneaux, Craig (2000): „Decision-Makers or Decision Takers? Military Missions and Civilian Control in Democratic South America", in: Armed Forces and Society, 26 (3), 413-436.

Rico, Aldo (1997): El retorno al proyecto nacional, Buenos Aires: Ediciones del MODIN.

Rouquié, Alain (1988): „Demilitarization and the Institutionalization of MilitaryDominated Politics in Latin America“, in: O’Donnell, Guillermo/Schmitter, Philippe C./Whitehead, Laurence (Hrsg.): Transitions From Authoritarian Rule. Comparative Perspectives, Baltimore/London: The Johns Hopkins University Press, 108-136.

Stepan, Alfred (1988): Rethinking Military Politics: Brazil and the Southern Cone, Princeton: Princeton University Press.

Varas, Augusto (1990): „Las relaciones cívico-militares en un marco democrático“, in: Goodman, Louis/Mendelson, Johanna/Rial, Juan (Hrsg.): Los Militares y la Democracia, Montevideo: Peitho.

Zagorski, Paul (1992): Democracy vs. National Security: Civil-Military Relations in Latin America, Boulder: Lynne Rienner Publishers. 